\title{
Elena Goun
}

\author{
Bioluminescence lights her way to measure glucose uptake in vivo, and why a chemist travels outside her comfort zone.
}

$\mathrm{T}$ he probe ${ }^{18} \mathrm{~F}-\mathrm{FDG}$ is used with positron emission tomography. It's pretty much the only in vivo probe for quantifying glucose uptake, says Elena Goun, a chemist on the faculty of the Swiss Federal Institute of Technology (EPFL) in Lausanne. But ${ }^{18} \mathrm{~F}$-FDG must be produced in a cyclotron, it's radioactive and therefore must be transported securely, its half-life is around 120 minutes and it's pricey. She wondered about an alternative.

"I find it very exciting to try and push myself to work on the frontiers," she says. Biological problems are difficult and "way outside of my comfort zone." Her father died of cancer, which adds to her motivation to address pressing biomedical challenges. Her PhD advisor and "scientific father," Stanford University chemist Paul Wender, taught her to start from a biological question and then work on the chemistry. This has become "a lifelong inspiration," she says. "That's what I want my lab to do."

Goun and her team have developed and validated BiGluc, a probe that harnesses bioluminescence to quantitatively measure glucose uptake and flux. Glucose is one of the body's major metabolites and plays a role in many conditions, including cancer and metabolic disease. BiGluc is stable and can be stored for years, she says.

In the lab's comparative tests, BiGluc was as sensitive as ${ }^{18} \mathrm{~F}$-FDG. She hopes other labs will use it, given how many glucose probes work in vitro but fail in vivo. Goun believes the new approach can be applied beyond glucose for quantifying uptake of nucleic acids, amino acids, lipids or carbohydrates. "This is our dream: we want to develop a platform to look for metabolite uptake using optical imaging," she says.

BiGluc luminesces from deep inside an animal's body and needs no excitation light. With fluorescent probes, it's this excitation light that can lead to a noisy signal, because it makes many biomolecules fluoresce.

"The beautiful part about bioluminescence is that there's no background," she says.

In fireflies, bioluminescence results from the interaction of the enzyme luciferase and the substrate luciferin. Here, the team wanted tunable bioluminescence: the light was to shine when a cell took up glucose.

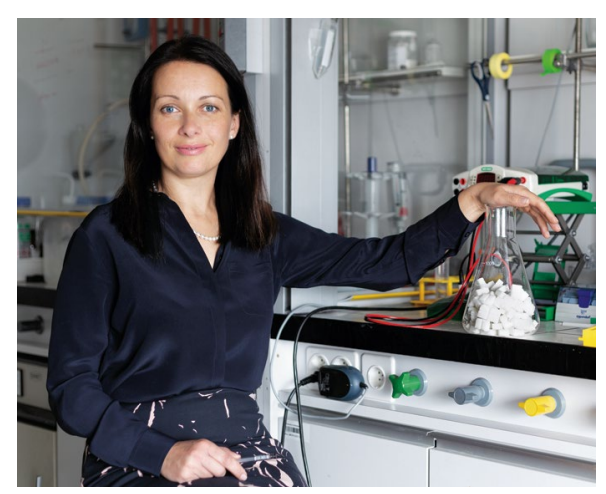

Elena Goun. Credit: E. Budnikova

They linked luciferin to the sugar in different ways and injected the molecules. But there was no light. Glucose transporters are highly selective, if not to say "very finicky", says Goun. They had shut the cellular door to the modified glucose as if to say: "No, I don't recognize it as a glucose anymore."

They eventually modified glucose with azide to equip it with a 'click' handle, a technique developed in the lab of Goun's postdoctoral fellowship advisor Stanford chemist Carolyn Bertozzi. After cellular uptake, the modified sugar reacts with a secondary reagent, which 'uncages' the luciferin, and bioluminescence results that's proportional to the amount of modified glucose taken up into the cell.

Other tinkering involved making the azide reaction strong enough for the secondary reaction to occur and enable bright luminescence. There was a twopart injection process, the timing of which also had to be worked out. The team had to assure that the animal's physiology was not perturbed and that the probe was not cleared too quickly from the animal's body. In vivo probes have to work "on the level of the whole organism," she says.

In Goun's lab, there are chemists, chemical engineers and biologists from whom she feels she learns every day. She also deeply enjoys teaching classes in chemical biology and medicinal chemistry, where she encourages students to think about how to apply their knowledge to find ways to treat disease better than currently possible.

When she first joined EPFL, she was the full-time manager of a research and exchange program with Russia, her native country, which she found fun and gratifying. She enjoys interacting with people and building bridges to other disciplines. "I think that's where I get the majority of my ideas," she says. When she's not in the lab, she spends time with family and enjoys hiking and running. A few days a week, she grabs her dog for a run on a trail on the banks of Lake Geneva.

\section{"I find it very exciting to try and push myself to work on the frontiers."}

"Elena is a translational-scientist rock star," says Wender. As he learned after the fact, Goun had spent evenings at a company he co-founded to access equipment and work on her own. One day, he recalls, they had chatted about inventing a new way to measure the thickness of a mouse's inflamed ear. An hour later, she returned to Wender's office with a colleague from Stanford's biodesign center. "To the best of my knowledge, she did not know this person but apparently walked through the center asking for someone with relevant expertise and then convinced him to engage in a collaboration," he says. "When she decides to do something, she really locks in." Her passion, creativity and ability to make things happen have led to a research program of impressive depth and breadth, where she is defining new frontiers where physics and the life sciences intersect, he says. "She is an incredible scholar, person and a force of nature."

Vivien Marx

Published online: 13 May 2019 https://doi.org/10.1038/s41592-019-0429-4

Reference

Maric, T. et al. Bioluminescent-based imaging and quantification of glucose uptake in vivo. Nat. Methods. https://doi.org/10.1038/s41592-019-0421-z (2019). 